\title{
Angiogenin elevates the invasive potential of squamous cell lung carcinoma cells through epithelial-mesenchymal transition
}

\author{
LI XU ${ }^{1,2^{*}}$, YAN YAN $^{1,3^{*}}$, XIANG XUE ${ }^{1}$, CHUN-GUANG LI $^{1}$, ZHI-YUN XU $^{1}$ and HE-ZHONG CHEN ${ }^{1}$ \\ ${ }^{1}$ Department of Cardiothoracic Surgery, Changhai Hospital, Second Military Medical University, \\ Shanghai; ${ }^{2}$ Department of Cardiothoracic Surgery, Nanjing Drum Tower Hospital, The Affiliated \\ Hospital of Nanjing University Medical School, Nanjing, Jiangsu; ${ }^{3}$ Cardiovascular Therapeutic Centre, \\ The 117 Hospital of the Chinese People's Liberation Army, Hangzhou, Zhejiang, P.R. China
}

Received April 23, 2016; Accepted June 7, 2016

DOI: $10.3892 /$ or.2016.5107

\begin{abstract}
Squamous cell carcinoma of the lung is one of the most aggressive cancers, and its aggressiveness is in part due to its intrinsic high rate of metastasis. Moreover, the process of epithelial-mesenchymal transition (EMT) appears to be involved in these neoplastic processes. Furthermore, EMT-type cells share many biological characteristics with the function of angiogenin (ANG) in squamous cell lung carcinoma. We conducted immunohistochemical analysis to detect the expression of ANG, E-cadherin, vimentin, $\mathrm{N}$-cadherin, $\beta$-catenin and TGF- $\beta 1$ in 60 cases of squamous cell lung carcinoma tissues. Western blot analysis was adopted to detect the protein expression levels of ANG and EMT markers. The effects of ANG on proliferation, migration and invasion of squamous cell lung carcinoma cells was analyzed by Cell Counting Kit-8, scratch assay and Transwell invasion chamber in order to reveal the role of ANG in the process of EMT in squamous cell lung carcinoma. The results revealed that ANG was aberrantly expressed in the squamous cell lung carcinoma specimens and was closely correlated with the differentiation of the cell lines. The expression of ANG was also significantly associated with metastasis and the stage of the squamous cell lung carcinoma cases. In addition, we validated that ANG influenced the expression of vimentin, E-cadherin, $\mathrm{N}$-cadherin, $\beta$-catenin and TGF- $\beta 1$ in
\end{abstract}

Correspondence to: Dr He-Zhong Chen or Dr Zhi-Yun Xu, Department of Cardiothoracic Surgery, Changhai Hospital, Second Military Medical University, 168 Changhai Road, Shanghai 200438, P.R. China

E-mail:drchenhz@163.com

E-mail: drxuzhiyun111@163.com

${ }^{*}$ Contributed equally

Abbreviations: ANG, angiogenin; EMT, epithelial-mesenchymal transition; EGFR, epidermal growth factor receptor; SQCLC, lung squamous cell carcinoma

Key words: squamous cell lung carcinoma, ANG, EMT, invasion, metastasis
SK-MES-1 cells. Most importantly, overexpression of ANG enhanced the migration and invasion of SK-MES-1 cells, while knockdown resulted in opposite effects. In the present study, we found that ANG plays an important role in EMT in squamous cell lung carcinoma and may be a valuable therapeutic target for squamous cell lung carcinoma.

\section{Introduction}

With the advancements in research on mutations in the epidermal growth factor receptor (EGFR) kinase, as well as fusions involving anaplastic lymphoma kinase (ALK), the treatment of patients with lung adenocarcinoma has experienced marked improvement $(1,2)$. Unfortunately, the therapeutic effect of these personalized treatment programs is less obvious in the second most common type of lung cancer, squamous cell lung carcinoma (SQCLC). Hence, it is necessary to identify other significant markers as new targets for SQCLC therapy.

Angiogenin (ANG), a 14,400 Da angiogenic ribonuclease of the RNase A superfamily, is upregulated in a variety of human cancers and has a 33\% amino acid identity and an overall homology of $56 \%$ to that of RNase-A (3). Angiogenin was once thought to promote cancer progression through its angiogenic activity. However, recent research has demonstrated that ANG can directly stimulate tumor cell proliferation and its expression and activity were found to be upregulated significantly in a variety of human tumors including breast, colorectal, kidney and pancreatic cancers, and this upregulation was found to be correlated with tumor progression and poor prognosis in patients (4). As a transcription factor, ANG cannot be detected in normal, non-endothelial cells (5), however, its expression in human cancer cells and human tumors has not been widely investigated.

More importantly, ANG is implicated in a wide range of biological functions including cell survival, growth, proliferation, migration, tube formation and tumor angiogenesis (6-8).

Epithelial-mesenchymal transition (EMT), a process in which epithelial cells lose or modify their apical-basal polarity and are converted to a mesenchymal phenotype, is a critical process during embryonic development and tumor metastasis $(9,10)$. The process of EMT is typically characterized 
by losing the expression of the epithelial markers, E-cadherin and $\beta$-catenin, which are transmembrane proteins essential for stable adherens junctions, and acquiring the expression of mesenchymal markers, such as $\mathrm{N}$-cadherin, vimentin and TGF- $\beta(11,12)$. We, therefore, hypothesized that ANG is an inducer of EMT in human squamous cell lung carcinoma and enhances the metastatic potential.

\section{Materials and methods}

Tissue collection. The cancerous and corresponding non-tumor normal specimens of SQCLC, freshly frozen for western blot analysis and qRT-PCR, were obtained from 50 patients who underwent lung cancer resection procedures at Changhai Hospital from October, 2012 to October, 2015. All cancerous and matching non-cancerous samples used for this study were provided by the Clinical and Experimental Pathology of the Research Centre of Changhai Hospital, Shanghai, China where initial H\&E staining and histologic diagnoses were performed after standard surgical primary tumor resection. This study was conducted in accordance with the Declaration of Helsinki and was approved by the Medical Ethics Committee of Changhai Hospital, with all patients providing written informed consent.

Cell culture. SK-MES-1 cells were obtained from the Cancer Cell Repository (Shanghai Cell Bank, Shanghai, China), and were maintained in Dulbecco's modified Eagle's medium (DMEM) supplemented with $10 \%$ heat-inactivated fetal bovine serum (Gibco-BRL) and antibiotics $(100 \mathrm{U} / \mathrm{ml}$ penicillin and $100 \mathrm{U} / \mathrm{ml}$ streptomycin; Hyclone Laboratories, Inc., Logan, $\mathrm{UT}, \mathrm{USA}$ ) at $37^{\circ} \mathrm{C}$ in a humidified atmosphere of $5 \% \mathrm{CO}_{2}$.

RNA interference and ANG overexpression. The ANG-specific short hairpin RNA adenovirus vector (shANG) and the negative control short hairpin RNA adenovirus vector (shScramble) were purchased from Genechem Co., Ltd. (Shanghai, China). The sequences of shANG were: sense strand 1: 5'-CCGCGGG ATGACAGATACTGTGAAGAGATTCACAGTATCTGTCA TCCCG-3' and antisense strand 1: 5'-AACGGGATGACAGA TACTGTGAATCTCTTCACAGTATCTGTCATCCCGC-3'; sense strand 2: 5'-CCGGATCCCAGGCTCGTTCTTTGGAG ACAAAGAACGAGCCTGGGATCC-3' and antisense strand 2: 5'-AAGGATCCCAGGCTCGTTCTTTGTCTCCAA AGAACGAGCCTGGGATCC-3'. An adenovirus vector co-expressing ANG (Ad-ANG) and a control empty adenovirus vector (Ad-Null) were constructed using AdEasy system (Genechem Co., Ltd., Shanghai, China).

Quantitative real-time reverse-transcription polymerase chain reaction ( $q R T-P C R)$. Total RNA was extracted and cDNA was synthesized using the PrimeScript RT Reagent kit (Takara Bio). SYBR Premix EX Taq (Takara Bio) was used for quantitative real-time polymerase chain reaction performed using a LightCycler ${ }^{\circledR} 480$ Real-Time PCR system (Roche Diagnostics). The primers for each gene were as follows: $18 \mathrm{~S}$ rRNA forward: 5'-CGGACACGGACAGGATTGAC-3'; 18S rRNA reverse: 5'-GCATGCCAGAGTCTCGTTCG-3'; ANG forward: 5'-GGACTTGTTCTGAGGCCGAG-3'; ANG reverse: 5'-CCAGCACGAAGACCAACAAC-3'. The amplifi- cation conditions were as follows: 1 cycle of $95^{\circ} \mathrm{C}$ for $30 \mathrm{sec}$ and 40 cycles of $95^{\circ} \mathrm{C}$ for $5 \mathrm{sec}$ followed by $60^{\circ} \mathrm{C}$ for $30 \mathrm{sec}$. The expression levels of the target genes relative to the control were determined using the $2^{-\Delta \Delta \mathrm{CT}}$ method. $18 \mathrm{~S}$ rRNA served as the internal control.

Western blot analysis. Western blot analyses were performed as previously described (13). The primary antibodies included: angiogenin (Sc-74528, Santa Cruz Biotechnology, Inc., 1:1,000), vimentin (ab92547, Abcam, 1:2,000), TGF- $\beta 1$ (ab92486, Abcam, 1:1,000), E-cadherin (ab40772, Abcam, 1:10,000) and $\beta$-catenin (60008-1-Ig, Proteintech, 1:2,000).

Immunohistochemistry. Immunohistochemical assay was performed as previously described (14). The primary antibodies used were: angiogenin (Sc-74528, Santa Cruz Biotechnology, Inc., 1:100), vimentin (ab92547, Abcam, 1:200), TGF- $\beta 1$ (ab92486, Abcam, 1:100), E-cadherin (ab40772, Abcam, 1:500), N-cadherin (ab12221, Abcam, 1:500), and $\beta$-catenin (ab32572, Abcam, 1:500).

Immunofluorescence. Paraformaldehyde-fixed SK-MES-1 cells were first incubated with primary antibodies at $4^{\circ} \mathrm{C}$ overnight. After being washed three times with PBS, the cells were incubated with secondary antibodies for $1 \mathrm{~h}$ at $37^{\circ} \mathrm{C}$. Nuclei were counterstained with 4',6-diamidino2-phenylindole (DAPI) for $2 \mathrm{~min}$. The primary antibodies used were angiogenin (Sc-74528, Santa Cruz Biotechnology, Inc., 1:1,000), E-cadherin (ab40772, Abcam, 1:500) and $\mathrm{N}$-cadherin (ab12221, Abcam, 1:500). The secondary antibodies used were Alexa Fluor 498-conjugated anti-rabbit IgG (1:400, Jackson ImmunoResearch).

Scratch assay. Cells were placed in a 24-well plate at an initial density of $1 \times 10^{5}$ cells/well. A uniform monolayer formed in 2-3 days. All wound-healing assays were performed in a serum-free medium. Thirty-six hours after infection, a micropipette tip was used to create a wound in the monolayer by scraping. Images were captured at $24 \mathrm{~h}$ after the scratch and the migrated cells were quantitated.

Invasion assay. The invasion assay was performed using Transwell insert chambers with a pore size of $8 \mu \mathrm{m}$ (Corning). The Transwell filter inserts were coated with Matrigel; $0.5 \times 10^{5}$ cells were seeded in serum-free medium in the upper chamber. After a $24-\mathrm{h}$ incubation at $37^{\circ} \mathrm{C}$, cells in the upper chamber were carefully removed with a cotton swab and the cells that had traversed the membrane were fixed, stained in a $0.1 \%$ crystal violet solution and counted.

Cell proliferation. Cell proliferation was analyzed using a Cell Counting Kit-8 (Beyotime, China). Twelve hours after being plated into a 96-well plate at a density of 2,000 cells/well the cells were transfected with an adenovirus. Next, the cells were incubated for $0,24,48$ and $72 \mathrm{~h}$; then, $10 \mu \mathrm{l} \mathrm{CCK-8} \mathrm{solution}$ was added to each well and the cultures were incubated at $37^{\circ} \mathrm{C}$ for $1 \mathrm{~h}$. Subsequently, the absorbance at $450 \mathrm{~nm}$ was measured.

Statistical analysis. All statistical analyses were performed using SPSS version 19.0. The quantitative data were first evalu- 
A

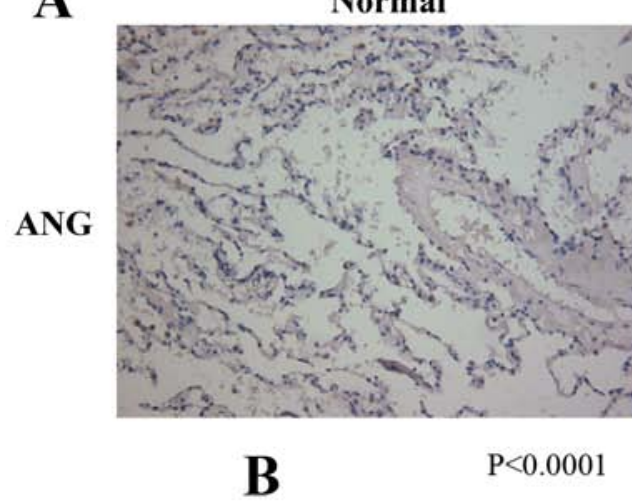

B

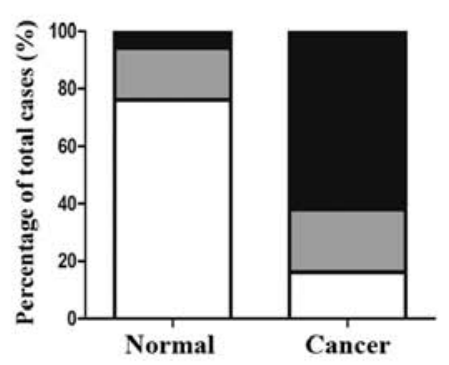

LG

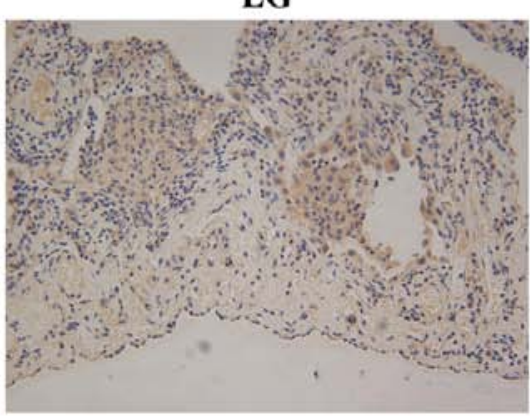

C
High $\square$ Negative

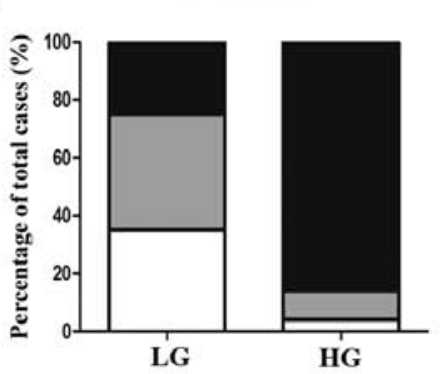

HG

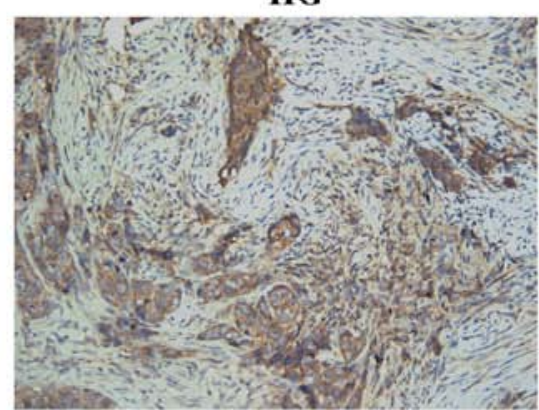

$\square$ High
$\square$ Low
$\square$ Negative

Figure 1. ANG is expressed in SQCLC tissues and is correlated with poor postoperative surgical-pathologic staging. (A) Immunohistochemical assays showing ANG expression in low-grade and high-grade SQCLC and normal lung tissues adjacent to tumors. (B) Percentage of normal and cancer cases with high, moderate and negative staining for ANG. (C) Percentage of low-grade and high-grade cases with high, low and negative staining for ANG. ANG, angiogenin; SQCLC, squamous cell lung carcinoma; LG, low-grade; HG, high-grade.

A
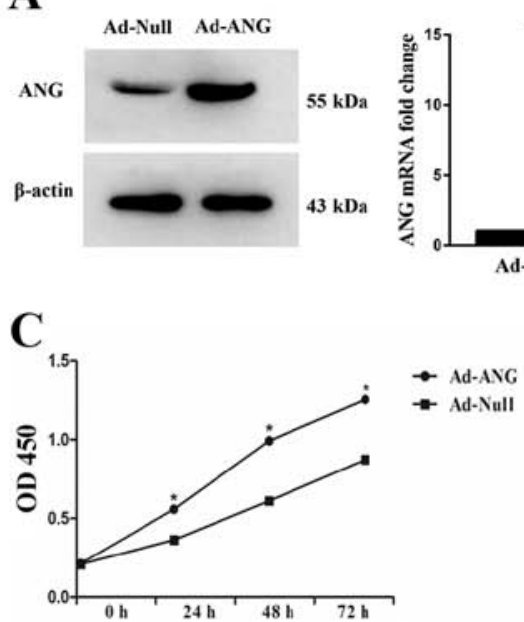

*

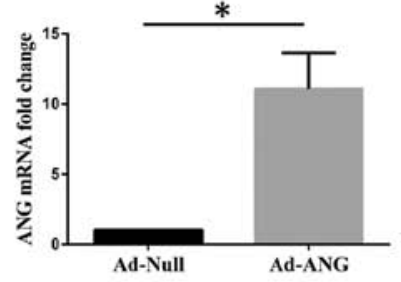

B

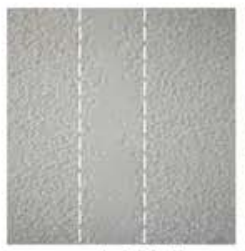

Ad-Null

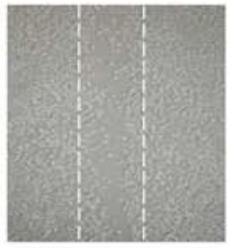

Ad-ANG

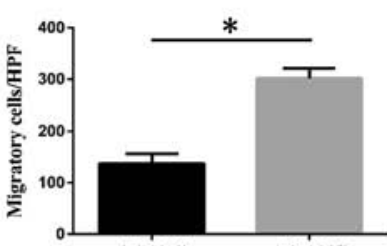

Ad-Null

Ad-ANG

D

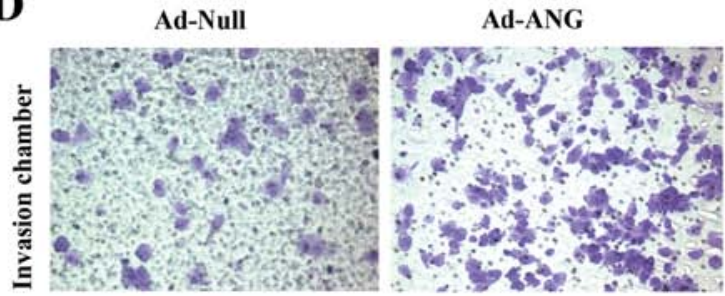

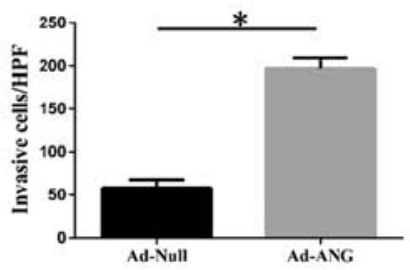

Figure 2. Upregulation of angiogenin (ANG) enhances the proliferation, migration and invasion of SK-MES-1 cells. (A) mRNA and protein levels of ANG were significantly enhanced in the Ad-ANG SK-MES- 1 cells ( $n=3$ for each cell group). Ad-Null served as the control. "P<0.05 vs. control. (B) Scratch assay was used to evaluate the migration capability of the SK-MES-1 cells in 2-dimensional space (magnification, x100). Ad-ANG SK-MES-1 cells showed a higher migration capability. Quantified data are presented as the number of migrated cells per high-power field (HPF) $24 \mathrm{~h}$ after scratch. Ad-Null served as the control. "P<0.05 vs. control. (C) Higher optical density at $450 \mathrm{~nm}$ in the CCK-8 assay was observed in the Ad-ANG SK-MES-1 cells at 24,48 and $72 \mathrm{~h}$, respectively. "P<0.01 vs. control. (D) Transwell invasion chamber assay was used to evaluate the invasion capability of the SK-MES-1 cells in 3-dimensional space (magnification, x200). Ad-ANG SK-MES-1 cells showed higher invasion capability. Quantified data are presented as the number of invasive cells per HPF. All experiments were carried out in triplicate. ${ }^{*} \mathrm{P}<0.01$ vs. control.

ated on whether they followed the normal distribution by the Shapiro-Wilk test. The data with a non-normal distribution were analyzed using the Kruskal-Wallis test. The data with a normal distribution were assessed using the Student's t-test. A P-value $<0.05$ was considered to be a statistically significant difference.

\section{Results}

$A N G$ is expressed in SQCLC and positively correlates with a high grade of malignancy of $S Q C L C$. Immunohistochemical assays showed that ANG was expressed in the SQCLC 
A
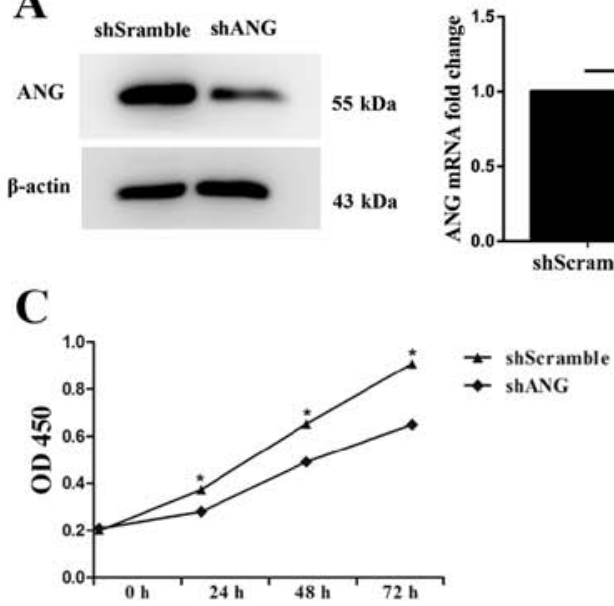

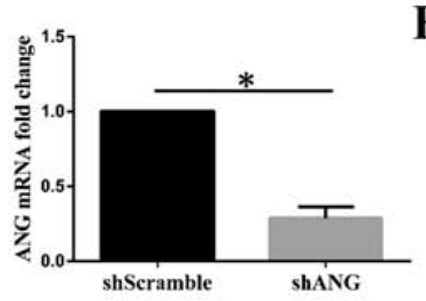

D
B

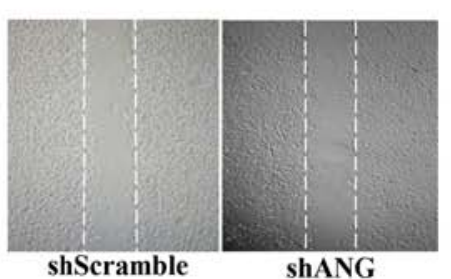

shScramble
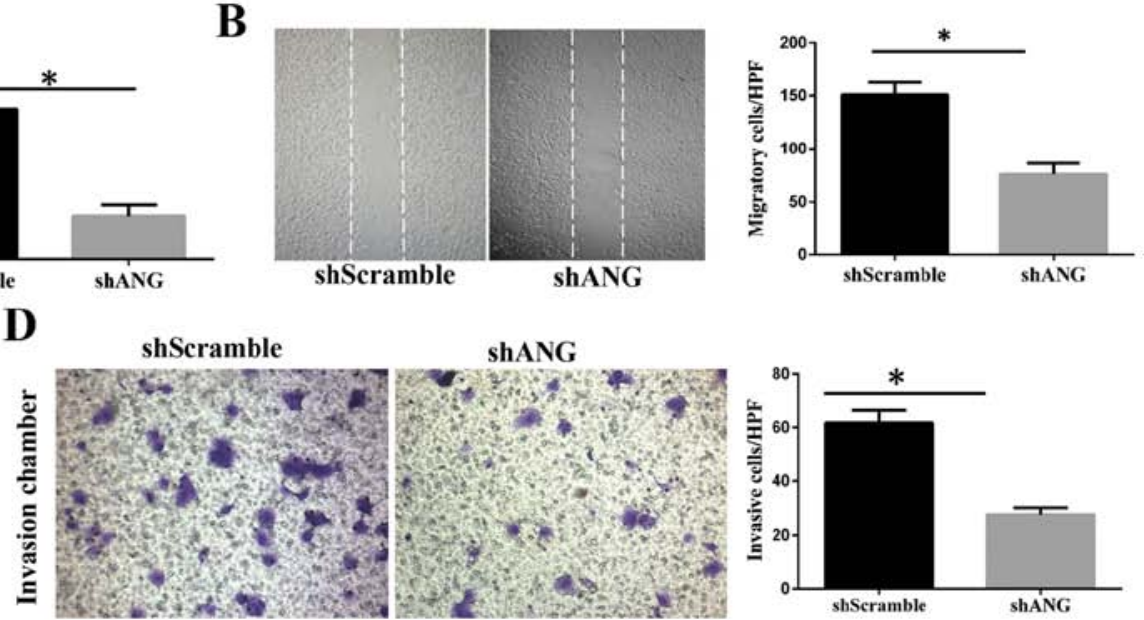

Figure 3. Knockdown of angiogenin (ANG) reduces the ability of the proliferation, migration and invasion of SK-MES-1 cells. (A) ANG protein and mRNA expression was decreased in the shANG SK-MES-1 cells, as compared with the shScramble SK-MES-1 cells ( $\mathrm{n}=3$ for each cell group). shScramble served as the control. " $\mathrm{P}<0.05$ vs. the control. (B) shANG SK-MES-1 cells showed a lower migration capability. Quantified data are presented as the number of migrated cells per high-power field (HPF) $24 \mathrm{~h}$ after being scratched. shScramble served as the control. " $\mathrm{P}<0.05 \mathrm{vs}$. the control. (C) shANG SK-MES-1 cells showed a lower proliferation capacity at 24,48 , and $72 \mathrm{~h}$, as compared with the shScramble SK-MES- 1 cells ( $\mathrm{n}=3$ for each cell group). ${ }^{*} \mathrm{P}<0.05 \mathrm{vs}$. the control. (D) Transwell invasion chamber assay was used to evaluate invasion capability of the SK-MES-1 cels in 3-dimensional space (magnification, $\mathrm{x} 200$ ). shANG SK-MES-1 cells showed lower invasion capability. Quantified data are presented as the number of invasive cells per HPF. All experiments were carried out in triplicate. $\mathrm{P}<0.05$ vs. the control.
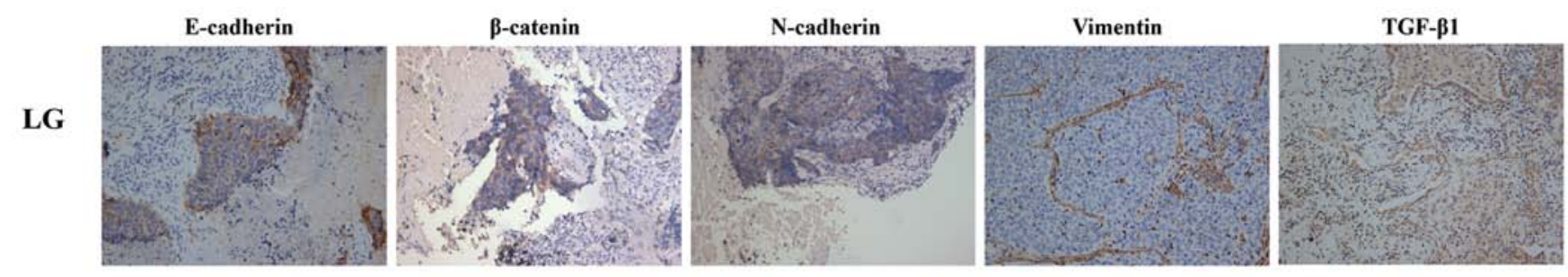

HG
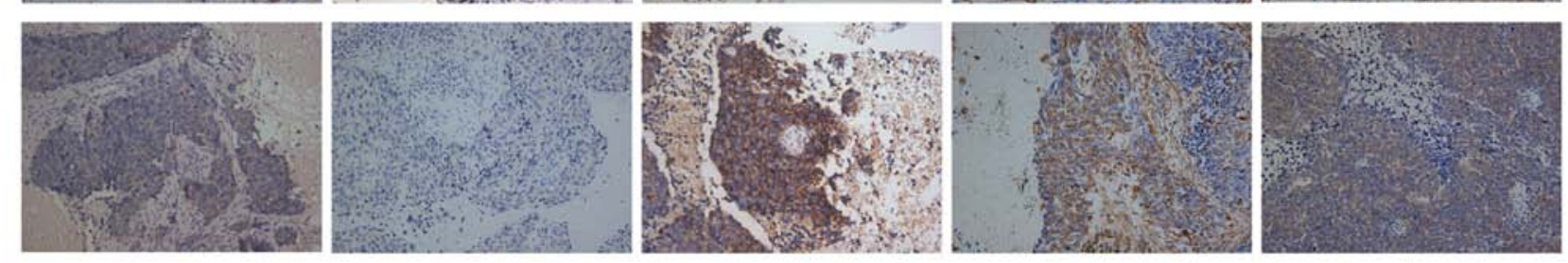

Figure 4. Immunohistochemical staining of mesenchymal and epithelial markers, including TGF- $\beta 1$, vimentin, N-cadherin, E-cadherin and $\beta$-catenin in SQCLC. Compared with low-grade SQCLC, epithelial markers including E-cadherin and $\beta$-catenin were significantly reduced, while mesenchymal markers including $\mathrm{N}$-cadherin, vimentin and TGF- $\beta 1$ were markedly enhanced in the high-grade SQCLC. SQCLC, squamous cell lung carcinoma; LG, low-grade; HG, high-grade.

tissues, while it was not detected in the normal lung tissues adjacent to tumors (Fig. 1A). As shown in Fig. 1B, ANG was significantly increased in the SQCLC tissues compared with that noted in the normal lung tissues $(\mathrm{P}<0.0001)$. Notably, high-grade SQCLC which indicates a higher postoperative surgical-pathologic staging exhibited the strongest ANG expression (Fig. 1A). Consistently, the difference in the patterns of ANG expression in low-grade and high-grade SQCLC was significant (low-grade vs. high-grade, $\mathrm{P}<0.0001$, Fig. 1C), which showed that a high percentage of high-grade SQCLC cases had strong ANG expression when compared with the expression in its low-grade counterpart. Collectively, our results indicated that ANG expression increased with the malignant grade of SQCLC.

Upregulation of ANG enhances proliferation, migration and invasion of SK-MES-1 cells in vitro. Since the local expression level of ANG was considerably increased in the SQCLC tissues, especially in the cancerous tissues from high-grade SQCLC patients where ANG expression was intensely positive, as compared with the adjacent normal tissue, we considered that ANG may play an important role in the development, metastasis and invasiveness of SQCLC. To investigate the effect of ANG on SQCLC, we used an in vitro system. Firstly, we effectively infected SK-MES-1 cells with Ad-ANG (Fig. 2A). Evaluation of cell proliferation was performed after infection using a CCK-8 assay kit. It was found that Ad-ANG SK-MES-1 cells manifested increased proliferation levels at 24, 48 and $72 \mathrm{~h}$ after infection (Fig. 2C). With regard to SK-MES-1 cell migration and invasion capability evaluation, scratch assay and invasion chamber assays were applied to assess the impact of ANG on these cell properties. Results showed that Ad-ANG SK-MES-1 cells presented not only significantly increased migration but 


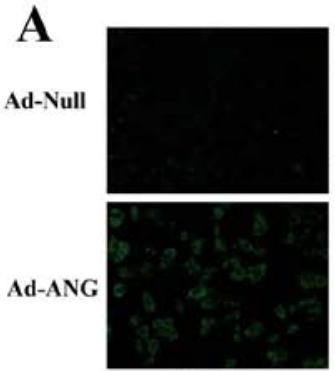

$\mathrm{N}$-cadherin
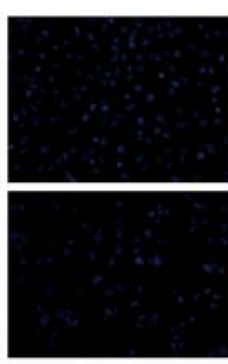

DAPI

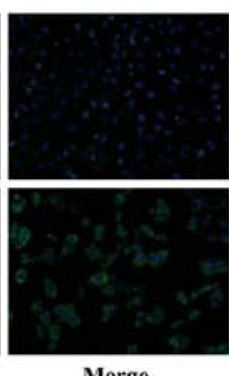

Merge

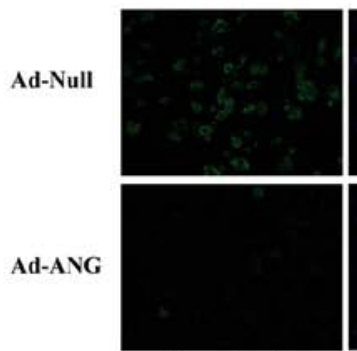

E-cadherin

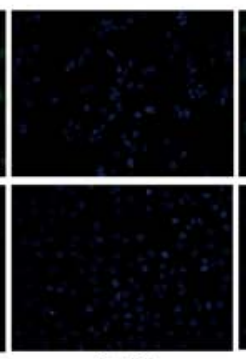

DAPI

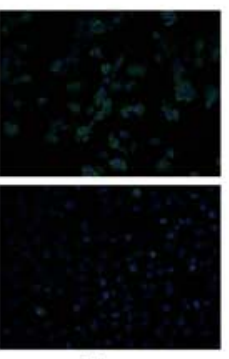

Merge
B

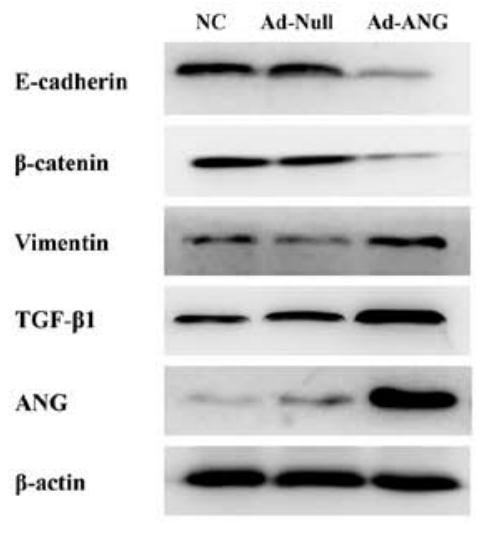

$135 \mathrm{kDa}$
$90 \mathrm{kDa}$
$55 \mathrm{kDa}$
$19 \mathrm{kDa}$
$55 \mathrm{kDa}$
$43 \mathrm{kDa}$
C

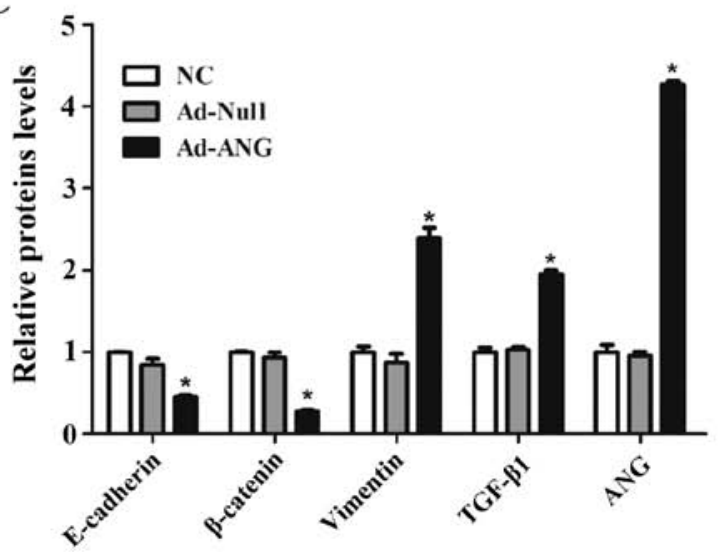

Figure 5. Overexpression of ANG induces EMT. (A) Expression levels of E-cadherin and N-cadherin as detected by immunofluorescence analysis. Nuclei were counterstained with DAPI (blue). The images are at a magnification of x200. (B and C) Western blot analysis showed that the expression levels of E-cadherin and $\beta$-catenin were downregulated and the expression levels of N-cadherin, vimentin and TGF- $\beta 1$ were upregulated in the Ad-ANG Sk-MES-1 cells. Data are expressed as the mean $\pm \mathrm{SD}$. ${ }^{*} \mathrm{P}<0.05$ vs. the control.

also invasion capability (Fig. 2B and D), as compared with the Ad-Null cells.

These results definitely indicated that ANG is an important regulator of proliferation, migration and invasion in SK-MES-1 cells.

Knockdown of $A N G$ reduces the ability of proliferation, migration and invasion of SK-MES-1 cells in vitro. We further used shANG to knock down ANG in SK-MES-1 cells. The cells were infected with shANG and cultured for $48 \mathrm{~h}$. SK-MES-1 cells infected with shScramble were used as a negative control. Compared with the shScramble SK-MES-1 cells, the expression level of ANG mRNA in the shANG cells was decreased by $70 \%$ (Fig. 3A). Western blot analysis results also confirmed lower expression level of ANG in the shANG SK-MES-1 cells (Fig. 3A). Furthermore, it was revealed that the shANG SK-MES-1 cells showed decreased proliferation capability (Fig. 3C). Meanwhile, decreased migration and invasion capacities were also observed in the shANG SK-MES-1 cells, as compared with the shScramble SK-MES-1 cells (Fig. 3B and D).

EMT is associated with the grade of malignancy of SQCLC. Given the fact that EMT contributes to metastasis and invasiveness of various cancers, we examined the expression of several EMT markers in both low-grade and high-grade SQCLC tissues. As shown in Fig. 4, compared with low-grade SQCLC, epithelial markers including E-cadherin and $\beta$-catenin were significantly reduced, while mesenchymal markers including $\mathrm{N}$-cadherin, vimentin and TGF- $\beta 1$ were markedly enhanced in the high-grade SQCLC, indicating that EMT may contribute to a metastatic and aggressive phenotype of SQCLC.

Overexpression of ANG upregulates the expression of mesenchymal markers but downregulates epithelial markers in the SK-MES-1 cell line. After showing that both ANG and EMT were positively correlated with a high grade of malignancy of SQCLC, we further tested whether ANG induces the metastatic and invasive phenotype of SQCLC by augmenting EMT. As shown in Fig. 5A, we found that the E-cadherin staining was decreased while $\mathrm{N}$-cadherin staining was increased in the Ad-ANG SK-MES-1 cells as compared with the Ad-Null cells. Furthermore, western blot analysis confirmed that the expression of vimentin and TGF- $\beta 1$ was significantly enhanced in the Ad-ANG cells. In contrast, Ad-ANG SK-MES-1 cells exhibited a lower level of protein expression of epithelial markers including E-cadherin and $\beta$-catenin, as compared with the Ad-Null and negative control groups (Fig. 5B and C, respectively; $\mathrm{P}<0.01)$. These data indicated that $\mathrm{ANG}$ triggers the process of EMT in SK-MES-1 cells.

\section{Discussion}

ANG, a $14.4 \mathrm{kDa}$ polypeptide, was first isolated from serumfree supernatants of a human adenocarcinoma cell line, HT-29 (15). In addition to angiogenic activity, ANG is also implicated in a variety of biological functions including cell 
growth, proliferation, migration and tube formation $(6,8,16)$. Of note, some studies have reported that ANG can undergo nuclear translocation to stimulate rRNA transcription in both cancer cells and cancer-associated endothelial cells (17). Recent research has shown that the expression and activity of ANG are upregulated in many human tumors including breast, kidney, colorectal and lung cancers (18). In the present study, immunohistochemical assays showed that ANG was expressed in SQCLC tissues, while it was not expressed in normal lung tissues adjacent to tumors, indicating that ANG is involved in the development of SQCLC, which is consistant with the finding that increased ANG is positively associated with the incidence of human tumors.

ANG has been reported as a member of the urokinase plasminogen activator receptor interactome that participates in the formation of plasmin and the migration of breast cancer cells, which are necessary for tumor metastasis and invasion (19). In a clinical study, researchers found that, among the markers representing different aspects of cancer vascular biology and exhibiting abnormal expression in colorectal cancer, ANG was the only index which was independently associated with the increasing stage of colorectal cancer according to the Dukes' and American Joint Committee on Cancer systems (20). Similarly, in patients with adenocarcinoma, our previous study showed that increased expression of ANG was correlated with vascular and pleural invasion as well as positive lymph node metastasis (6). Consistently, we found that increased expression of ANG was positively correlated with the high grade of SQCLC while knockdown of ANG abrogated proliferation, migration and invasion of SK-MES-1 cells. These results from clinical specimens and cultured squamous cell lung carcinoma cell lines supported the notion that ANG contributes to the invasive phenotype of squamous cell lung carcinoma cells.

Numerous studies have demonstrated that cell invasion during tumor progression may be dependent on the acquisition of EMT features (21). Evidence from clinical studies also suggests that poor survival of cancer patients and drug-resistance are associated with EMT phenotypes in malignant cancer cells (22). EMT is a complex process during which epithelial cells lose their polarity and cell-cell adhesion, exhibit enhanced cell-extracellular matrix adhesion, gain invasive properties and become mesenchymal-like cells. In both squamous cell lung carcinoma tissues and SK-MES-1 cells, we showed that high expression of ANG was positively correlated with the expression of mesenchymal markers but negatively associated with epithelial markers. These data suggest that ANG induces EMT in squamous cell lung carcinoma. The intermediate filament protein vimentin is an important marker of EMT and a critical regulator of mesenchymal cell migration. Induction of vimentin is associated with increased expression of Axl which enhanced the migratory activity of pre-malignant breast epithelial cells (23). Cell adhesion molecules are important to cancer invasion and metastasis. E-cadherin to $\mathrm{N}$-cadherin switch was found to promote cancer progression via TGF- $\beta$-induced EMT in extrahepatic cholangiocarcinoma (24) and is of strong importance in the progression of prostate cancer (25). In lung cancer cell line, H1650ER, N-cadherin expression was also reported to be upregulated and paralleled by the reduced expression of E-cadherin. Proliferation and invasion of H1650ER cells were inhibited by knockdown of N-cadherin (26). In accordance with these previous studies, our results indicated that overexpression of ANG led to the upregulation of vimentin, fibronectin and $\mathrm{N}$-cadherin and reduced expression of E-cadherin, suggesting that ANG contributes to invasion and metastasis of lung carcinoma by inducing EMT.

Many molecular and cell signaling pathways have been reported to participate in the EMT process. For instance, accumulating evidence indicates that the phosphatidylinositol 3-kinase (PI3K)/Akt/mTOR pathway plays an important role in EMT $(27,28)$. The MAPK/ERK pathway has also been demonstrated to be critical for many features of EMT including acquisition of invasive properties, enhanced MMP activity, and attenuation of adherens junctions. EMT mediated by TGF- $\beta$ also involves the activation of the MAPK/ERK pathway (29). In addition, research has shown that receptor activator of NF- $\mathrm{NB}$ (RANK) can induce EMT in human mammary epithelial cells and promotes oncogenesis and metastasis (30). Notably, correlation of ANG and these cell signaling pathways has been demonstrated in many studies. Overexpression of ANG can activate phosphorylation of downstream molecules of the PI3K/AKT/mTOR signaling pathway in bladder cancer cells (31). In cultured human umbilical vein endothelial cells, ANG induced the activation of both ERK1/2 and SAPK/JNK $(32,33)$. Moreover, ANG prevented stress-induced death of P19 embryonal carcinoma cells via upregulation of the Bcl-2 and NF- $\mathrm{B}$ pathways (34). Given these results from extensive studies, we speculate that ANG may induce EMT via a complex regulating network involving various cell signaling pathways.

In summary, this study suggests that ANG promotes the invasion and metastasis of SQCLC by enhancing proliferative, migratory and invasive ability of squamous cell carcinoma cells through the induction of EMT. Our results highlight the possibility that ANG may serve as a target for the treatment of squamous cell carcinoma of the lung.

\section{Acknowledgements}

This study was supported by the National Natural Science Foundation of China (nos. 81272592 and 81301829).

\section{References}

1. Paez JG, Jänne PA, Lee JC, Tracy S, Greulich H, Gabriel S, Herman P, Kaye FJ, Lindeman N, Boggon TJ, et al: EGFR mutations in lung cancer: Correlation with clinical response to gefitinib therapy. Science 304: 1497-1500, 2004.

2. Soda M, Choi YL, Enomoto M, Takada S, Yamashita Y, Ishikawa S, Fujiwara S, Watanabe H, Kurashina K, Hatanaka H, et al: Identification of the transforming EML4-ALK fusion gene in non-small-cell lung cancer. Nature 448: 561-566, 2007.

3. Li S and Hu GF: Angiogenin-mediated rRNA transcription in cancer and neurodegeneration. Int J Biochem Mol Biol 1: 26-35, 2010.

4. Nilsson UW, Abrahamsson A and Dabrosin C: Angiogenin regulation by estradiol in breast tissue: tamoxifen inhibits angiogenin nuclear translocation and antiangiogenin therapy reduces breast cancer growth in vivo. Clin Cancer Res 16: 3659-3669, 2010.

5. Ibaragi S, Yoshioka N, Kishikawa H, Hu JK, Sadow PM, Li M and Hu GF: Angiogenin-stimulated rRNA transcription is essential for initiation and survival of AKT-induced prostate intraepithelial neoplasia. Mol Cancer Res 7: 415-424, 2009.

6. Yuan Y, Wang F, Liu XH, Gong DJ, Cheng HZ and Huang SD: Angiogenin is involved in lung adenocarcinoma cell proliferation and angiogenesis. Lung Cancer 66: 28-36, 2009. 
7. Ivanov P, Emara MM, Villen J, Gygi SP and Anderson P: Angiogenin-induced tRNA fragments inhibit translation initiation. Mol Cell 43: 613-623, 2011.

8. Li S and Hu GF: Emerging role of angiogenin in stress response and cell survival under adverse conditions. J Cell Physiol 227 2822-2826, 2012.

9. Polyak K and Weinberg RA: Transitions between epithelial and mesenchymal states: Acquisition of malignant and stem cell traits. Nat Rev Cancer 9: 265-273, 2009.

10. Mani SA, Guo W, Liao MJ, Eaton EN, Ayyanan A, Zhou AY, Brooks M, Reinhard F, Zhang CC, Shipitsin M, et al: The epithelial-mesenchymal transition generates cells with properties of stem cells. Cell 133: 704-715, 2008 .

11. Zeisberg $\mathrm{M}$ and Neilson EG: Biomarkers for epithelial-mesenchymal transitions. J Clin Invest 119: 1429-1437, 2009.

12. Kokkinos MI, Wafai R, Wong MK, Newgreen DF, Thompson EW and Waltham M: Vimentin and epithelial-mesenchymal transition in human breast cancer-observations in vitro and in vivo. Cells Tissues Organs 185: 191-203, 2007.

13. Kurozumi A, Kato M, Goto Y, Matsushita R, Nishikawa R, Okato A, Fukumoto I, Ichikawa T and Seki N: Regulation of the collagen cross-linking enzymes LOXL2 and PLOD2 by tumor-suppressive microRNA-26a/b in renal cell carcinoma. Int J Oncol 48: 1837-1846, 2016.

14. Hu K, Tian Y, Du Y, Huang L, Chen J, Li N, Liu W, Liang Z and Zhao L: Atrazine promotes RM1 prostate cancer cell proliferation by activating STAT3 signaling. Int J Oncol 48: 2166-2174, 2016.

15. Fett JW, Strydom DJ, Lobb RR, Alderman EM, Bethune JL, Riordan JF and Vallee BL: Isolation and characterization of angiogenin, an angiogenic protein from human carcinoma cells. Biochemistry 24: 5480-5486, 1985.

16. Miyake M, Goodison S, Lawton A, Gomes-Giacoia E and Rosser CJ: Angiogenin promotes tumoral growth and angiogenesis by regulating matrix metallopeptidase- 2 expression via the ERK1/2 pathway. Oncogene 34: 890-901, 2015.

17. Li L, Pan XY, Shu J, Jiang R, Zhou YJ and Chen JX: Ribonuclease inhibitor up-regulation inhibits the growth and induces apoptosis in murine melanoma cells through repression of angiogenin and ILK/PI3K/AKT signaling pathway. Biochimie 103: 89-100, 2014.

18. Shu J, Huang M, Tian Q, Shui Q, Zhou Y and Chen J: Downregulation of angiogenin inhibits the growth and induces apoptosis in human bladder cancer cells through regulating AKT/mTOR signaling pathway. J Mol Histol 46: 157-171, 2015.

19. Dutta S, Bandyopadhyay C, Bottero V, Veettil MV, Wilson L, Pins MR, Johnson KE, Warshall C and Chandran B: Angiogenin interacts with the plasminogen activation system at the cell surface of breast cancer cells to regulate plasmin formation and cell migration. Mol Oncol 8: 483-507, 2014.

20. Ramcharan SK, Lip GY, Stonelake PS and Blann AD: Angiogenin outperforms VEGF, EPCs and CECs in predicting Dukes' and AJCC stage in colorectal cancer. Eur J Clin Invest 43: 801-808, 2013.

21. Makker A and Goel MM: Tumor progression, metastasis, and modulators of epithelial-mesenchymal transition in endometrioid endometrial carcinoma: An update. Endocr Relat Cancer 23: R85-R111, 2016.
22. Mitra A, Mishra L and Li S: EMT, CTCs and CSCs in tumor relapse and drug-resistance. Oncotarget 6: 10697-10711, 2015.

23. Vuoriluoto K, Haugen H, Kiviluoto S, Mpindi JP, Nevo J, Gjerdrum C, Tiron C, Lorens JB and Ivaska J: Vimentin regulates EMT induction by Slug and oncogenic H-Ras and migration by governing Axl expression in breast cancer. Oncogene 30: 14361448,2011

24. Araki K, Shimura T, Suzuki H, Tsutsumi S, Wada W, Yajima T, Kobayahi T, Kubo N and Kuwano H: E/N-cadherin switch mediates cancer progression via TGF- $\beta$-induced epithelial-tomesenchymal transition in extrahepatic cholangiocarcinoma. $\mathrm{Br}$ J Cancer 105: 1885-1893, 2011.

25. Gravdal K, Halvorsen OJ, Haukaas SA and Akslen LA: A switch from $\mathrm{E}$-cadherin to $\mathrm{N}$-cadherin expression indicates epithelial to mesenchymal transition and is of strong and independent importance for the progress of prostate cancer. Clin Cancer Res 13: 7003-7011, 2007.

26. Zhang X, Liu G, Kang Y, Dong Z, Qian Q and Ma X: N-cadherin expression is associated with acquisition of EMT phenotype and with enhanced invasion in erlotinib-resistant lung cancer cell lines. PLoS One 8: e57692, 2013.

27. Berg A, Hoivik EA, Mjøs S, Holst F, Werner HM, Tangen IL, Taylor-Weiner A, Gibson WJ, Kusonmano K, Wik E, et al: Molecular profiling of endometrial carcinoma precursor, primary and metastatic lesions suggests different targets for treatment in obese compared to non-obese patients. Oncotarget 6: 1327-1339, 2015.

28. Hipp S, Walch A, Schuster T, Losko S, Laux H, Bolton T, Höfler $\mathrm{H}$ and Becker KF: Activation of epidermal growth factor receptor results in snail protein but not mRNA overexpression in endometrial cancer. J Cell Mol Med 13: 3858-3867, 2009.

29. Edme N, Downward J, Thiery JP and Boyer B: Ras induces NBT-II epithelial cell scattering through the coordinate activities of Rac and MAPK pathways. J Cell Sci 115: 2591-2601, 2002.

30. Palafox M, Ferrer I, Pellegrini P, Vila S, Hernandez-Ortega S, Urruticoechea A, Climent F, Soler MT, Muñoz P, Viñals F, et al: RANK induces epithelial-mesenchymal transition and stemness in human mammary epithelial cells and promotes tumorigenesis and metastasis. Cancer Res 72: 2879-2888, 2012.

31. Peng Y, Li L, Huang M, Duan C, Zhang L and Chen J: Angiogenin interacts with ribonuclease inhibitor regulating PI3K/AKT/mTOR signaling pathway in bladder cancer cells. Cell Signal 26: 2782-2792, 2014.

32. $\mathrm{Xu} \mathrm{Z}$, Monti DM and Hu G: Angiogenin activates human umbilical artery smooth muscle cells. Biochem Biophys Res Commun 285: 909-914, 2001

33. Liu S, Yu D, Xu ZP, Riordan JF and Hu GF: Angiogenin activates Erk1/2 in human umbilical vein endothelial cells. Biochem Biophys Res Commun 287: 305-310, 2001.

34. Li S, Yu W, Kishikawa H and Hu GF: Angiogenin prevents serum withdrawal-induced apoptosis of P19 embryonal carcinoma cells. FEBS J 277: 3575-3587, 2010. 